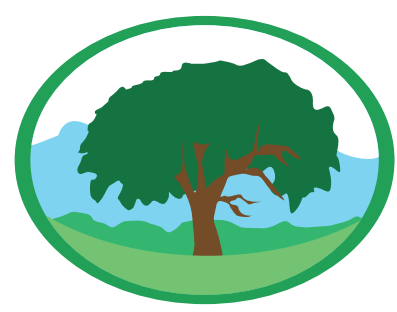

\title{
Filmes finos de nanotubos de carbono de parede múltipla preparados via spray
}

RODRIGUES, M. R. ${ }^{1}$; OSORIO, A. G. ${ }^{2}$; BERGMANN, C. P. ${ }^{3}$

${ }^{1}$ Mestranda no Programa de Pós-Graduação em Engenharia de Minas, Metalúrgica e de Materiais, Escola de Engenharia, UFRGS/Porto Alegre-RS.

${ }^{2}$ Profa. Dra., Programa de Pós-Graduação em Ciência e Engenharia de Materiais, Centro de Desenvolvimento Tecnológico, UFPel/Pelotas-RS.

${ }^{3}$ Prof.Dr., de Pós-Graduação em Engenharia de Minas, Metalúrgica e de Materiais, Escola de Engenharia, UFRGS/Porto Alegre-RS.

Palavras-chave: nanotubos de carbono, transmitância, ferromagnético

\begin{abstract}
Resumo
Neste estudo foram fabricados filmes finos de nanotubos de carbono de parede múltipla depositados via spray. Os nanotubos de carbono foram sintetizados por decomposição térmica de ferroceno, utilizando sílica como substrato. Através de espectroscopia Raman, observou-se as bandas $D, G$ e G', não sendo possível identificar as bandas da Região de Respiração Radial, o que permite caracterizar os nanotubos de carbono como de parede múltipla. Foi comprovada a capacidade ferromagnética do material através de magnetômetro de amostra vibrante. Os nanotubos de carbono foram dispersos em água com auxílio do surfactante dodecilbenzenossulfonato de sódio para aumento da molhabilidade. Os parâmetros de deposição otimizados foram $0,1 \mathrm{mg} / \mathrm{mL}$ de nanotubos de carbono, temperatura de $150{ }^{\circ} \mathrm{C}$, distância de $150 \mathrm{~mm}$ do substrato até o bico aspersor, pressão entre 1 e 2 bar e tempo de deposição entre 10 e 40 s. Após as deposições, as superfícies dos filmes foram observadas por microscopia óptica e a transmitância foi verificada por espectroscopia UV-visível. Os resultados indicaram que os filmes são heterogêneos e rugosos, apresentam redução da transmitância com o aumento do tempo de deposição e redução da espessura com o aumento da pressão.
\end{abstract}

\section{Thin films of multi-walled carbon nanotubes prepared by spray}

Key words: carbon nanotubes, transmittance, ferromagnetic

\begin{abstract}
In this study, thin films of multi-walled carbon nanotubes were manufactured by spray deposition. Carbon nanotubes were synthesized by thermal decomposition of ferrocene using silica as substrate. Through Raman spectroscopy, the bands D, G and G' were observed, as well as no Radial Respiration Region bands, evidencing the presence of multiple wall carbon nanotubes. The ferromagnetic capacity of the material was demonstrated through a vibrating sample magnetometer. The carbon nanotubes were dispersed in water with the aid of the sodium dodecylbenzenesulfonate surfactant to increase wettability. The optimized deposition parameters were $0.1 \mathrm{mg} / \mathrm{mL}$ of carbon nanotubes, $150{ }^{\circ} \mathrm{C}$ of temperature, $150 \mathrm{~mm}$ of distance, pressure between 1 and 2 bar and time of deposition between 10 and $40 \mathrm{~s}$. After the surface depositions, the surfaces of the films were observed by optical microscope and the transmittance was verified by UV-visible spectroscopy. The results indicated that the films are heterogeneous and rough, showing a transmittance decrease as the deposition time increases, as well as a thickness reduction as the pressure increases.
\end{abstract}




\section{INTRODUÇÃO}

Existem inúmeras pesquisas sobre nanotubos de carbono (NTCs), pois estes apresentam um conjunto de propriedades que comparadas com as de outros materiais, os tornam muito interessantes para aplicaçôes em Engenharia. A síntese de NTCs de parede múltipla pode ser realizada através de mais de uma técnica, entretanto, a técnica de deposição química de vapor catalisada (CCVD) possui um aparato simples e possibilita uma maior variação dos parâmetros (Osorio, 2013). A síntese é realizada utilizando o ferroceno $\left(\mathrm{Fe}\left(\mathrm{C}_{5} \mathrm{H}_{5}\right)_{2}\right)$, um organometálico, como catalisador e precursor que é arrastado por um gás inerte até um substrato onde ocorre a deposiçáo e o crescimento dos NTCs (Osorio \& Bergmann, 2013). Os filmes finos são lâminas delgadas com espessura de ordem nanométrica e micrométrica. Esses são muito utilizados como componentes semicondutores e em revestimentos ópticos como filmes condutores transparentes (FCT) (Ansón-Casaos et al., 2015). A deposição de filmes de NTCs, além de possuírem as propriedades habituais dos NTCs e possíveis propriedades como FCT, visa à utilização em dispositivos magnéticos, pelas partículas de ferro encapsuladas nos nanotubos. As primeiras tentativas de deposição de FCT de NTC foram feitas por evaporação de solvente (Meitl et al., 2004). Posteriormente, outras técnicas de deposição de filmes começaram a ser estudadas para os NTC, como spin coating, dipcoating (Mirri et al., 2012), rodcoating (Dan et al., 2009) e deposição por spray (Meng et al., 2014; Abdelhalim et al., 2013). Apesar de já registrado na literatura, a deposição por spray para obtenção de filmes de NTCs não foi muito explorada. Esta técnica foi, então, escolhida para esse trabalho por ter um aparato simples, de baixo custo e pelo controle de parâmetros como tempo de deposiçáo, temperatura e distância, entre outros. O presente trabalho tem como finalidade sintetizar NTCs e obter filmes finos preparados via spray, analisando parâmetros de deposição e caracterizar os filmes assim obtidos via transmitância e microscopia óptica.

\section{MATERIAIS E MÉTODOS}

\section{Material}

Para a síntese dos nanotunos de carbono de parede múltipla (NTCPM) e obtenção dos filmes finos, foram utilizados os seguintes produtos químicos: Ferroceno 98\% $\left(\mathrm{Fe}\left(\mathrm{C}_{5} \mathrm{H}_{5}\right)_{2}\right)$, Sigma Aldrich; e surfactante dodecilbenzenossulfonato de sódio (SDBS), Sigma Aldrich, massa molecular 348,48 g. Sílica $\left(\mathrm{SiO}_{2}\right)$, Aerosil 200 (Evonik Ind.). Água miliQ tipo 1 (Milisul), Direct-Q 3UV.

\section{Síntese e caracterização dos nanotubos de carbono}

A síntese dos NTCPMs foi realizada através da técnica CCVD, utilizando ferroceno $\left(\mathrm{Fe}\left(\mathrm{C}_{5} \mathrm{H}_{5}\right)_{2}\right)$ como catalisador (Esconjauregui et al, 2009) e precursor, argônio como gás inerte e sílica $\left(\mathrm{SiO}_{2}\right)$, com área superficial de $200 \mathrm{~m}^{2} / \mathrm{g}$, como substrato (Osorio \& Bergmann, 2013).A caracterização do material obtido foi realizada através de espectroscopia Raman modelo Renishaw inVia Spectrometer System ( $\mathbb{\nabla}=532 \mathrm{~nm})$. $\mathrm{E}$ a caracterização das propriedades magnéticas dos NTCPMs foi realizada por magnetômetro de amostra vibrante (MAV), modelo EZ9 MicroSense, analisando o ponto de saturação máxima da magnetização no sentido positivo e negativo da histerese magnética após aplicação do campo magnético, avaliando o ponto de magnetização de saturação e magnetização de remanência.

\section{Dispersão dos nanotubos de carbono}

A dispersão dos NTCPMs foi feita com auxílio de surfactante dodecilbenzenossulfonato de sódio (SDBS). A solução de SDBS $1 \% \mathrm{~m} / \mathrm{m}$ foi feita com água miliQ e solubilizada com auxílio de ultrassom de banho por $10 \mathrm{~min}$ (Meng et al., 2014). Após, os NTCPMs foram adicionados na solução de SDBS, e para auxiliar na dispersáo foi colocado em ultrassom de ponteira por $36 \mathrm{~min}$ com banho de gelo, pulso de $30 \mathrm{~s} / 59 \mathrm{~s}$ e intervalo de $5 \mathrm{~min}$ a cada $12 \mathrm{~min}$ de funcionamento do equipamento para reduzir ao máximo o aquecimento da dispersão e do equipamento. A molhabilidade da dispersão perante o substrato de vidro foi analisada pelo ângulo de contato, utilizando 
um goniômetro Digidrop (Labometric), modelo LBDX.

\section{Deposição dos filmes de nanotubos de carbono}

A deposição dos filmes de NTCPM foi realizada por spray usando lâminas de vidro como substrato. Os parâmetros de deposição dos filmes foram variados quanto a sua concentração de NTCPM, distância de deposição, pressão, vazão da bomba peristáltica, temperatura e tempo de deposição. As concentraçóes analisadas foram de 0,$1 ; 0,2 ;$ e $0,4 \mathrm{mg} / \mathrm{mL}$ de NTCPM. As distâncias do bico aspersor até o substrato analisadas foram 100, 150 e $200 \mathrm{~mm}$. As pressôes utilizadas foram 1 e 2 bar. A vazáo da bomba peristáltica foi de 0,6 ; 1 ; e $2 \mathrm{~mL} / \mathrm{min}$. A temperatura utilizada foi 100 e $150{ }^{\circ} \mathrm{C}$. A espessura do filme foi variada através do tempo de deposição, 10, 20 e 40 s. Após a deposição dos filmes, foram feitas imagens e análise de sua espessura em microscópio óptico Olympus BX41MLED, pela observação do topo e do fundo da amostra, utilizando as marcaçóes do foco fino do microscópio. A transmitância dos filmes foi analisada na faixa do UV-Vis, 200 a $800 \mathrm{~nm}$ no equipamento Cary 7000 Agilent Technologies.

\section{RESULTADOS E DISCUSSÃO}

\section{Caracterização dos nanotubos de carbono}

Espectroscopia Raman. A análise por espectroscopia Raman (Figura 1) permitiu identificar três picos em regiôes características que indicam a presença de NTCPM (Osorio \& Bergmann, 2013). A banda $\mathrm{D}$, em torno de $1350 \mathrm{~cm}^{-1}$, indica que quanto maior a sua intensidade maior a desordem e defeitos da estrutura. A banda G, em torno de $1570 \mathrm{~cm}^{-1}$, é um modo de alta frequência, associado às ligaçóes $s p^{2}$. A banda G', em torno de $2690 \mathrm{~cm}^{-1}$, é um modo de segunda ordem, associado à estrutura do grafite e também como harmônico da banda D (Lobo et al., 2005; Bokobza \& Zhang, 2012).

Propriedades magnéticas. A histerese mostrada na Figura 2 indica os pontos de saturação máxima do campo magnético, no sentido positivo e negativo, como sendo aproximadamente 630 Oe de

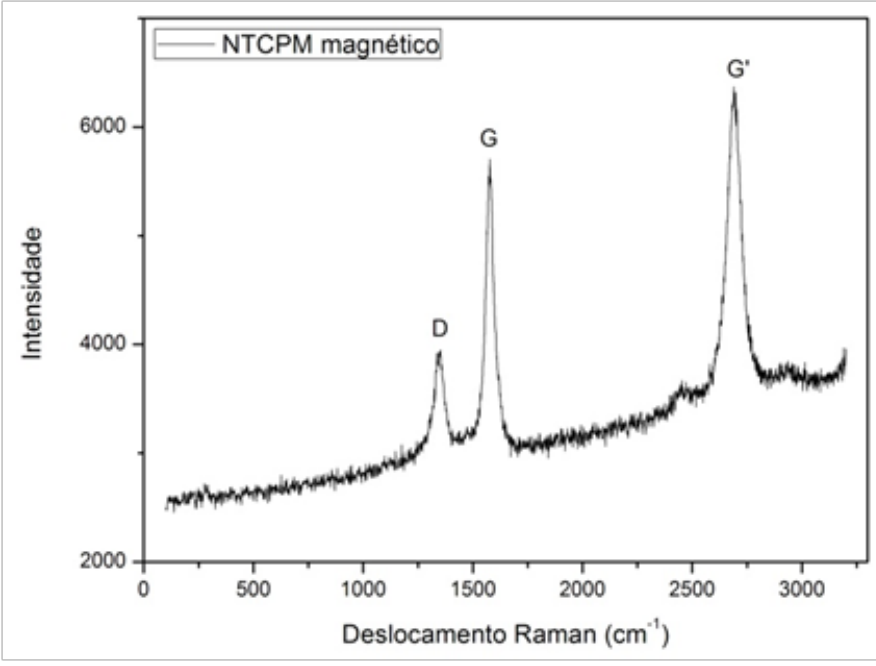

Figura 1: Espectro de Raman dos NTCPMs sintetizados

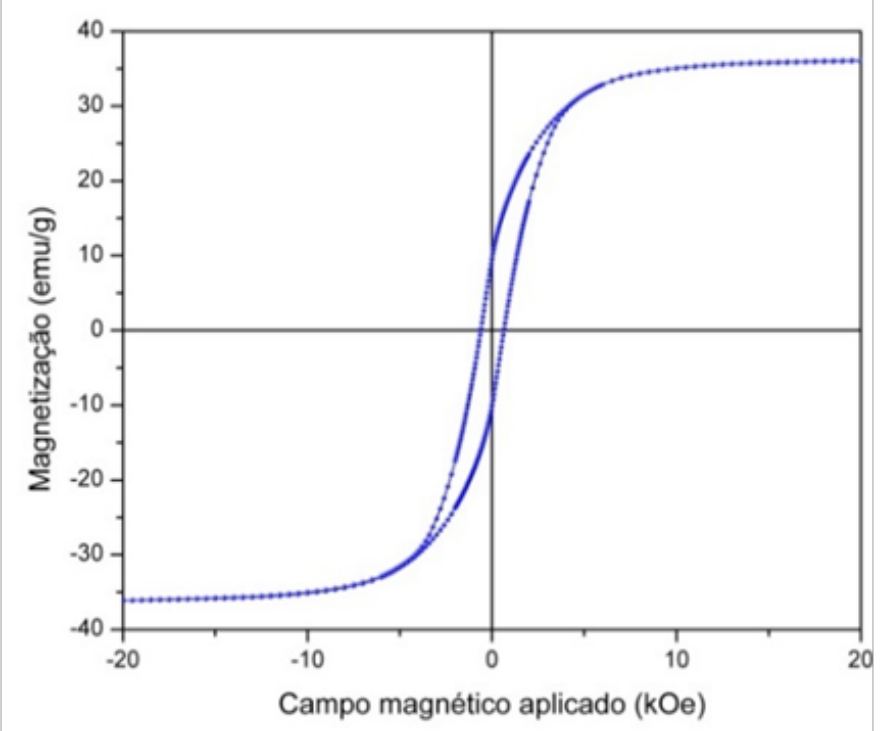

Figura 2: Histerese de magnetização do pó de NTCPM

coercividade média, e uma magnetização de $30 \mathrm{emu} / \mathrm{g}$. Também indica, através da magnetização remanente, aproximadamente $12 \mathrm{emu} / \mathrm{g}$, que o material possui comportamento ferromagnético proveniente das partículas de ferro encapsuladas no interior dos NTCPMs. O ferro possui uma coercividade de apenas 0,9 Oe, ao comparar com o valor de 630 Oe, encontrado para os NTCPM através do MAV, podese perceber um aumento significativo da coercividade (Osorio et al., 2013).

\section{Análise de molhabilidade}

A molhabilidade indica a capacidade do líquido em manter contato com a superfície sólida do substrato. Para verificar a molhabilidade, foram realizados testes 
de ângulo de contato entre o substrato e as dispersôes de NTCPM em solução de SDBS e água miliQ (NTCPM/SDBS). Foram realizadas análises das três diferentes dispersões de NTCPM/SDBS, 0,1;0,2 e $0,4 \mathrm{mg} / \mathrm{mL}$ e o valor do ângulo de contato ficou entre 15 e $22^{\circ}$, que indica um aumento significativo da molhabilidade quando comparada com a água, que possui ângulo de contato de aproximadamente $70^{\circ}$ (Gao et al., 2013; Meng et al., 2014). A Figura 3 mostra uma das imagens coletadas no teste de ângulo de contato, para a dispersão de $0,4 \mathrm{mg} / \mathrm{mL}$, onde obteve-se um ângulo de $17,5^{\circ}$.

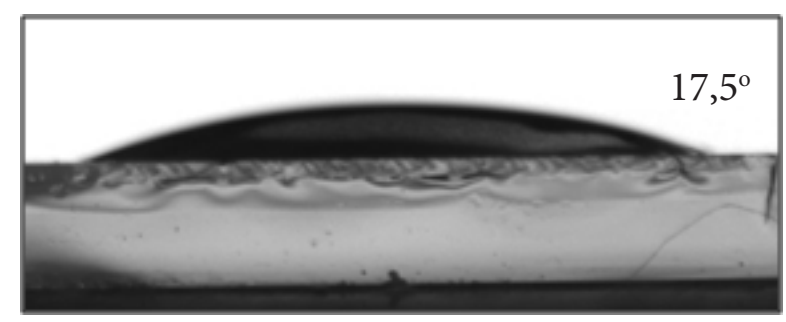

Figura 3: Imagem do ângulo de contato da dispersão $0,4 \mathrm{mg} / \mathrm{mL}$ de NTCPM/SDBS

\section{Otimização do processo de deposição e caracterização dos filmes de nanotubos de carbono}

A otimização dos parâmetros da deposição do filme de NTCPM foi realizada levando em consideração imagens do microscópio óptico e transmitância. As vazóes de 1 e $2 \mathrm{~mL} / \mathrm{min}$ da bomba peristáltica foram testadas e descartadas já no início da otimização dos parâmetros, pois havia uma perda significativa de amostra e a formação de um filme excessivamente espesso. A vazão da bomba peristáltica foi entáo reduzida para $0,6 \mathrm{~mL} / \mathrm{min}$, para a obtenção de filmes com menor espessura. A distância de $100 \mathrm{~mm}$ do bico aspersor e do substrato, nas diferentes concentraçôes de dispersão e na pressão de 1 e 2 bar, resultaram em filmes com aglomerados de NTCPM que podiam ser vistos a olho. Com o aumento da distância de aspersão para 200 mm, os filmes apresentaram-se mais homogêneos, mas houve uma perda de precursor, pois o spray abrangia uma área além da do substrato. A distância de aspersão de $150 \mathrm{~mm}$ nas pressóes de 1 e 2 bar e nas diferentes concentraçôes de dispersão possibilitou a obtenção de filmes homogêneos (sem aglomeração de NTCPMs), com um bom recobrimento da superfície do substrato, sem perdas significativas de precursor.
As concentraçóes de 0,4 e $0,2 \mathrm{mg} / \mathrm{mL}$ formaram um filme espesso, mas havia uma dificuldade em manter os NTCPMs dispersos dentro da mangueira da bomba peristáltica, provocando entupimento no aerógrafo e formando filmes heterogêneos. A concentração de $0,1 \mathrm{mg} / \mathrm{mL}$ resultou em uma maior estabilidade da dispersão e uma formação de filmes mais homogêneos. Foram testadas as temperaturas de deposição de $100 \mathrm{e}$ $150{ }^{\circ} \mathrm{C}$. Na temperatura de $100{ }^{\circ} \mathrm{C}$, após o início da deposição, verificou-se uma redução na temperatura do substrato, que por sua vez foi responsável por impedir o alcance da temperatura de evaporação da água, resultando em filmes com marcas de gotas a olho nu. Portanto, a temperatura de deposição ideal foi considerada $150{ }^{\circ} \mathrm{C}$, pois a evaporaçáo da água pôde ser garantida.

Assim, adotou-se $150 \mathrm{~mm}$ de distância do bico aspersor até o substrato; a concentração da dispersão de NTCPM/SDBS como sendo $0,1 \mathrm{mg} / \mathrm{mL}$; temperatura de $150{ }^{\circ} \mathrm{C}$; e a vazão da bomba peristáltica sendo 0,6 $\mathrm{mL} / \mathrm{min}$. Com esses parâmetros foram obtidos filmes variando a pressáo (1 e 2 bar) e o tempo de deposição $(10,20$ e 40 s). A Figura 4 mostra as análises de transmitância realizadas nos filmes obtidos utilizandose esses parâmetros. Os testes 1, 2 e 3 foram realizados a 1 bar, variando o tempo de deposição em 10, 20 e 40 s. Já os testes 4 , 5 e 6 foram realizados a 2 bar, variando o tempo de deposição da forma anterior. Ao analisar os filmes obtidos nos primeiros 3 testes (Figura 4), podese perceber que o filme menos concentrado teve uma maior transparência, como é esperado, e à medida que o tempo de deposiçáo aumenta (consequentemente aumentando a quantidade de material depositado), a transparência diminui. Os filmes de 4 a 6 também seguiram essa mesma tendência. É importante ressaltar que os filmes com pressão de 2 bar (testes 4, 5 e 6) tiveram uma maior transparência, levando em consideração os respectivos testes em 1 bar (testes 1, 2 e 3), indicando que a diferença de pressão resulta em um filme mais transparente no comprimento de onda analisado.

A Figura 5 mostra algumas imagens de microscópio óptico dos filmes de NTCPM. Por essas imagens, pode-se notar que há formação de pequenas gotas nos filmes, o que é esperado pela técnica de deposição 


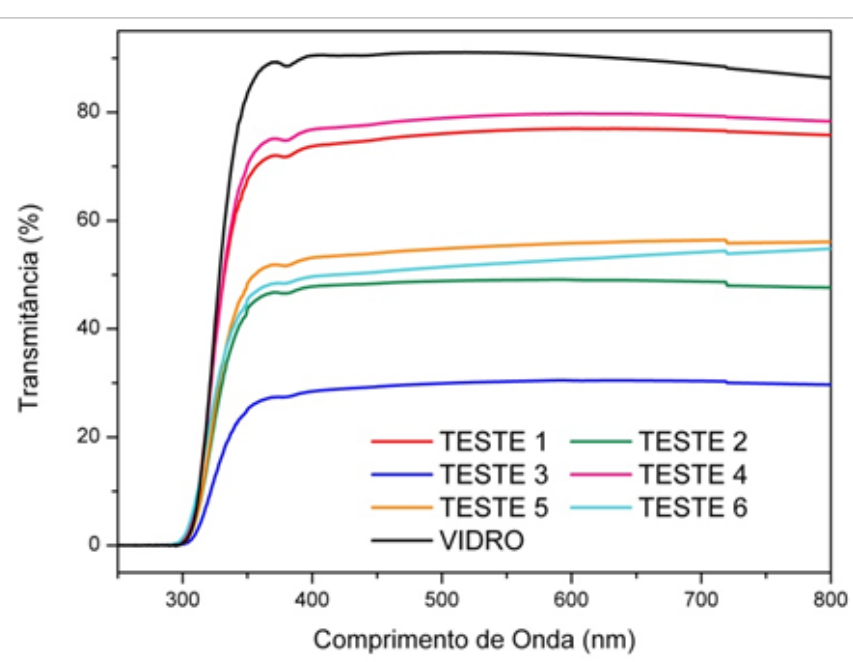

Figura 4: Variação da média da transmitância dos filmes de NTCPMs depositados via spray

utilizada. Importante ressaltar que as gotas formadas possuem uma característica distinta, já descrita na literatura, de o NTC ficar concentrado apenas na borda (Majumder et al., 2010). Os filmes com tempo de deposição de 10 e $20 \mathrm{~s}$ ficaram menos rugosos e mais transparentes que os de $40 \mathrm{~s}$, o que já era esperado já que a espessura dos filmes aumenta com o aumento do tempo de deposição.

Teste $1(100 \mathrm{x})$

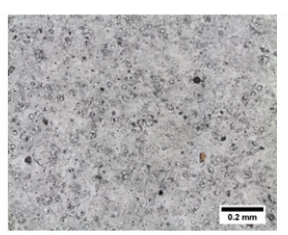

Teste $2(100 \mathrm{x})$

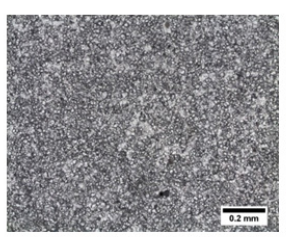

Teste $5(100 \mathrm{x})$

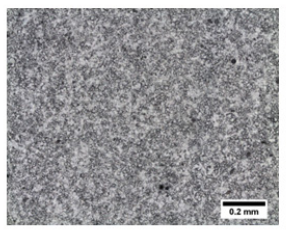

Figura 5: Imagens por microscopia óptica dos filmes de NTCPM depositados via spray

Para analisar a espessura dos filmes foram realizados testes em microscópio óptico. A Tabela 1 mostra a espessura média para cada filme de NTCPMs depositado via spray. Cada filme foi riscado com um material de dureza menor que a do substrato. A calibração do foco fino do microscópio óptico foi realizada utilizando a referência do rugosímetro. Inicialmente, utilizou-se o ajuste do foco fino para distinguir a diferença de espessura do topo do padrão até o vale do mesmo (Harris, 1985). Após, foi traçado um perfil do padrão no rugosímetro e então foram comparados os valores do gráfico de rugosidade com o valor da espessura encontrado através do foco fino. A seguir à calibraçáo, mediu-se a espessura em 10 pontos diferentes do filme e então calculou-se a média dos valores, como sendo a espessura média de cada filme. Importante ressaltar que a aquisição dos valores foi realizada após a regiáo de efeito de borda, causada pelo risco.

Tabela 1: Espessura média dos filmes de NTCPM depositados via spray realizada em microscópio óptico

\begin{tabular}{|l|l|}
\hline Filme & Espessura média $(\mu \mathrm{m})$ \\
\hline Teste 1 & 1,5 \\
\hline Teste 2 & 2,5 \\
\hline Teste 3 & 3 \\
\hline Teste 4 & 1 \\
\hline Teste 5 & 1,5 \\
\hline Teste 6 & 1,5 \\
\hline
\end{tabular}

Os valores de espessura corroboraram com os valores de transmitância. $\mathrm{O}$ aumento do tempo de deposição resulta em aumento da espessura dos filmes. Para os três primeiros testes (1, 2 e 3 ), com pressão igual a 1 bar, o valor da espessura foi maior que para os seus respectivos testes com pressão igual a 2 bar $(4,5$ e 6). Comparando os testes 4 e 5 , nota-se uma diferença considerável de transmitância para uma diferença de 0,5 $\mu \mathrm{m}$ na espessura média. Já a espessura média dos testes 5 e 6 foram iguais, enquanto que a transmitância teve uma leve diferença. Como a sensibilidade do método utilizado para medir a espessura é micrométrica, os testes 5 e 6 podem ter diferenças nanométricas em sua espessura, o que ocasionaria a diferença discreta na transmitância. Portanto, há evidências de que a pressão varia a espessura dos filmes. Ainda, pode-se esperar uma diferença da homogeneização do filme, o que resultaria em valores diferentes de transmitância, para uma mesma espessura. 


\section{CONCLUSÃO}

As análises de Raman das bandas D, G e G' indicaram características de NTCPM nos produtos sintetizados. O gráfico de histerese magnética dos NTCPM indicou que estes possuem comportamento ferromagnético, o que era esperado, já que a síntese realizada utiliza ferroceno como catalisador e precursor, e um aumento significativo da coercividade em relação à do ferro. Constatou-se pela análise de transmitância e imagens de microscopia óptica que foi possível otimizar os parâmetros de deposição, sendo $150 \mathrm{~mm}$ a distância de aspersão adotada (do substrato até o bico aspersor), concentração de $0,1 \mathrm{mg} / \mathrm{mL}$ de NTCPM, temperatura de $150{ }^{\circ} \mathrm{C}$ e vazáo da bomba de $0,6 \mathrm{~mL} / \mathrm{min}$, em 10 e $20 \mathrm{~s}$ de deposição. Através da transmitância e da análise de espessura, pode-se observar um possível padrão de redução na espessura dos filmes com o aumento da pressão de deposição.

\section{AGRADECIMENTOS}

Agradecimentos aos laboratórios LAM do Instituto de Física da UFRGS e ao CNPq pelo apoio financeiro.

\section{REFERÊNCIAS}

ABDELHALIM, A.; ABDELLAH, A.; SCARPA, G.; LUGLI, P. Fabrication of carbon nanotube thin films on flexible substrates by spray deposition and transfer printing. Carbon, v. 61, p. 7279, 2013.

ANSÓN-CASAOS, A.; MIS-FERNÁNDEZ, R.; LÓPEZALLED, C. M.; ALMENDRO-LÓPEZ, E.; HERNÁNDEZFERRER, J.; GONZÁLEZ-DOMÍNGUEZ, J. M.; MARTÍNEZ, M. T. Transparent conducting films made of different carbon nanotubes, processed carbon nanotubes, and graphene nanoribbons. Chemical Engineering Science, v. 138, p. 566-574, 2015.

BOKOBZA, L.; ZHANG, J. Raman spectroscopic characterization of multiwall carbon nanotubes and of composites. eXPRESS Polymer Letters,v.6, n.7,p. 601-608, 2012.
DAN, B.; IRVIN, G. C.; PASQUALI, M. Continuous and scalable fabrication of transparent conducting carbon nanotube films.ACS Nano, v. 3, p. 835-843, 2009.

ESCONJAUREGUI, S; WHELAN, C.M.; MAEX, K. The reasons why metals catalyze the nucleation and growth of carbon nanotubes and other carbon nanomorphologies. Carbon, v. 47, p. 659-669, 2009.

Gao, J.; Wang, W. Y.; Chen, L. T.; Cui, L. J.; Hu, X. Y.; Geng, H. Z. Optimizing processes of dispersant concentration and post-treatments for fabricating single-walled carbon nanotube transparent conducting films. Applied Surface Science, v. 277, p. 128-33, 2013.

HARRIS, R. M. Light microscopic depth measurements of thick sections. Journal of Neuroscience Methods, v. 14, p. 97-100, 1985.

LOBO, A.O.; MARTIN, A.A.; ANTUNES, E.F.; TRAVAAIROLDI, V.J.; CORAT, E.J. Caracterização de materiais carbonosos por espectroscopia Raman. Revista Brasileira de Aplicaçóes de Vácuo, v. 24, n. 2, p. 98-103, 2005.

MAJUMDER, M.; RENDALL, C.; LI, M.; BEHABTU, N.; EUKEL, J.A.; HAUGE, R.H.; SCHMIDT, H.K.; PASQUALI, M. Insights into the physics of spray coating of SWNT films. Chemical Engineering Science, v. 65, p. 2000-2008, 2010.

MEITL, M. A.; ZHOU, Y.; GAUR, A.; JEON, S.; USREY, M. L.; STRANO, M. S.; ROGERS, J. A. Solution casting and transfer printing single-walled carbon nanotube films. Nano Letters, v. 4, p. 1643-1647, 2004.

MENG, Y.; XU X.; LI, H.; WANG, Y.; DING, E.; ZHANG, Z.; GENG, H. Optimisation of carbon nanotube ink for largearea transparent conducting films fabricated by controllable rodcoating method. Carbon, v. 70, p. 103-110, 2014.

MIRRI, F.; MA, A. W. K.; HSU, T. T.; BEHABTU, N.; EICHMANN, S. L.; YOUNG, C. C.; TSENTALOVICH, D. E.; PASQUALI, M. High-performance carbon nanotube transparent conductive films by scalable dip coating. ACS Nano, v. 6, p. 9737-9744, 2012.

OSORIO, A.G. Obtenção de nanotubos de carbono por decomposição térmica de ferroceno e caracterização de sua morfologia e comportamento magnético. Porto Alegre: UFRGS, 2013. Tese doutorado.

OSORIO, A.G.; BERGMANN, C.P. Effect of surface area of substrates aiming the optimization of carbon nanotube production from ferrocene. Applied Surface Science, v. 264, p. 794-800, 2013.

OSORIO, A. G.; PEREIRA, L. G.; DA CUNHA, J. B. M.; BERGMANN, C. P. Controlling the magnetic response of carbon nanotubes filled withiron-containing material. Materials Research Bulletin, v. 48, p. 4168-4173, 2013. 